\title{
A IMPORTÂNCIA DO USO DA DOSE CORRETA NA ADUBAÇÃO NITROGENADA DE TIFTON 85
}

\author{
Nédio Luís Patzlaff ${ }^{1}$, Alisson Perin Zulpo², Danieli Simonetti Rossi ${ }^{3}$
}

\begin{abstract}
${ }^{1}$ Engenheiro Agrônomo, Mestre em Produção e Sanidade Animal; ${ }^{2}$ Engenheiro Agrônomo, Especialista em Agronegócios; ${ }^{3}$ Engenheira Agrônoma, Mestre em Desenvolvimento Regional; ${ }^{1,2,3}$ Extensionista Rural - Empresa de Pesquisa Agropecuária e Extensão Rural de Santa Catarina - Epagri
\end{abstract}

RESUMO: O estudo avaliou as respostas produtivas (massa verde - MV e massa seca - MS), a taxa de acúmulo de forragem - TAF, e a capacidade de suporte - CS da pastagem perene de verão Tifton 85 submetida à restrição ou excesso de nitrogênio em relação à dose recomendada. O delineamento experimental foi o inteiramente casualizado, com três doses de N: "meia", "correta" e "dobro" e quatro repetições em cada tratamento. Após um corte de uniformização, aplicou-se $\mathrm{N}$ na forma de ureia de acordo com os tratamentos, e aguardou-se 33 dias até os cortes de amostragem, tempo que a pastagem levou para atingir uma altura média de aproximadamente $25-28 \mathrm{~cm}$. Utilizou-se um quadrado de peças de PVC encaixadas com $0,25 \mathrm{~m}^{2}$, lançado aleatoriamente na parcela, para delimitar a área a ser cortada em cada amostragem. Os dados foram submetidos à testes de homogeneidade e normalidade, análise de variância e teste de comparação de médias por Tukey para os dados paramétricos e, para os não paramétricos, teste de Kruskal Wallis e de Wilcoxon $(p<0,03)$ no programa $R$. Houve influência dos tratamentos sobre todos os parâmetros avaliados, sendo que o tratamento "correta" foi superior em produção de MV, MS, TAF e CS. Já o teor de MS (\%) foi maior no tratamento com restrição de N (meia).

Palavras-chave: bovinocultura, Cynodon spp., manejo de pastagens

\section{THE IMPORTANCE OF USING THE RIGHT DOSE OF NITROGEN IN FERTILIZATION OF TIFTON 85}

ABSTRACT: The study evaluated the productive responses (green mass - MV and dry mass - MS), the forage accumulation rate - TAF, and the carrying capacity - CS 
of the perennial summer pasture Tifton 85 submitted to the restriction or excess of nitrogen $(N)$ in relation to the recommended dose. The experimental design was completely randomized, with three doses of $N$ : "half", "correct" and "double" and four replicates in each treatment. After a uniformation cut, $N$ was applied in the form of urea according to the treatments, and it was waited 33 days until the sampling cut, which time the pasture took to reach an average height of approximately $25-28 \mathrm{~cm}$. A square of PVC pieces fitted with $0.25 \mathrm{~m} 2$ was used, randomly launched on the pasture, to delimit the area to be cut in each sampling. Data were submitted to homogeneity and normality tests, analysis of variance and test of comparison of means by Tukey for parametric data and, for non-parametric ones, Kruskal Wallis and Wilcoxon test $(p<0.03)$ in the $R$ program. The treatments influenced all evaluated parameters, and the "correct" treatment was superior in the production of MV, MS, TAF and CS. The DM content (\%) was higher in the treatment with $N$ restriction (half).

Keywords: cattle breeding, Cynodon spp, pasture management.

\section{INTRODUÇÃO}

No Brasil, o volume de leite de vaca negociado em 1997 foi de 10,5 bilhões de litros. Já em 2018, passou para 24,4 bilhões, com destaque para a região Sul, respondendo por mais de $30 \%$ desse total, evidenciando a importância dessa atividade para as propriedades rurais dessa região (IBGE, 2020).

Sistemas de produção de leite que baseiam a dieta dos animais prioritariamente em pastagens reduzem a exposição à riscos econômicos e tornam as propriedades mais resilientes, quando comparados à sistemas de produção cuja dieta é baseada em milho e soja, pois o preço das commodities sofrem forte influência do mercado. Ainda assim, existem vazios forrageiros e, de acordo com Patzlaff et al. (2020), a reserva forrageira é responsável por equilibrar a oferta de alimento nesses períodos.

A pecuária leiteira catarinense está calcada em vacas criadas soltas a campo, com as mais diversas espécies forrageiras. Uma das que tem se destacado é o capim Tifton 85 (Cynodon spp.), desenvolvido em Coastal Plain Experiment Station 
(USDA - University of Geórgia), no sul do Estado da Geórgia (PREMAZZI \& MONTEIRO, 2002)

De acordo Hill et al. (1996) e Pedreira (1996), a capacidade do Tifton 85 de produzir elevados volumes com boa qualidade, o tornam uma ótima alternativa como forrageira. Para Alvim et al. (1998), os rizomas que a espécie possui lhe conferem boa resistência à seca e ao frio. Pode-se inferir que essa característica botânica também contribui para uma maior resistência ao pisoteio animal.

A adubação em pastagens, prática amplamente difundida, se torna essencial quando se pretende elevar os níveis produtivos das espécies forrageiras e sua capacidade de suporte animal (FAGUNDES et al., 2011).

No cotidiano da atividade, adubações sem prévia análise de solo ainda são habituais entre os pecuaristas. Percebe-se uma impressão empírica equivocada de que as respostas são diretamente proporcionais às quantidades de adubo utilizadas ou, ainda, sob 0 argumento de economizar, alguns produtores reduzem a quantidade de $\mathrm{N}$. Esse comportamento observado motivou a realização do presente estudo, com vistas a gerar resultados técnico/científicos que permitam contrapor as justificativas dessa prática, ainda comum.

Os estudos que testam doses de $\mathrm{N}$ raramente mencionam a dose adequada para aquela espécie naquela condição específica de solo em que está sendo testada. Geralmente são usadas doses numéricas crescentes, o que impossibilita saber se as respostas obtidas refletem o comportamento de uma planta que está idealmente nutrida ou não.

Diante disso, objetivou-se avaliar as respostas produtivas do Tifton 85 (Cynodon spp.) submetida ao uso da dose adequada de $\mathrm{N}$, comparado às respostas obtidas mediante restrição ou excesso desse nutriente.

\section{MATERIAL E MÉTODOS}

A pesquisa foi realizada nos meses de março e abril de 2019, em propriedade rural situada em Lindóia do Sul/SC, com sede nas coordenadas UTM 399797,55 "O" 
e 7008948,75 "S" e altitude de $751 \mathrm{~m}$. As normais climatológicas para o município, de acordo com o INMET (2020), correspondem à temperatura média anual de $19^{\circ} \mathrm{C}$, precipitação pluviométrica média anual de $1.950 \mathrm{~mm}$ e insolação média anual de 2.300 horas. A propriedade dispõe de um sistema de piquetes manejados sob pastejo racional. A área experimental foi alocada em um dos piquetes com Tifton 85, cujo solo é classificado como cambissolo, com as seguintes características: Argila = $25 \% ; \mathrm{SMP}=6,4 ; \mathrm{pH}=6,1 ; \mathrm{P}=84,7 \mathrm{mg} \mathrm{L}^{-1} ; \mathrm{K}=300 \mathrm{mg} \mathrm{L}^{-1} ; \mathrm{MO}^{2}=3,7 ; \mathrm{Ca}^{2+}=6,6$ cmol(c) $\mathrm{L}^{-1} ; \mathrm{Mg}^{2+}=3,0 \mathrm{cmol}(\mathrm{c}) \mathrm{L}^{-1} ; \mathrm{Al}^{3+}=0 \mathrm{cmol}(\mathrm{c}) \mathrm{L}^{-1} ; \mathrm{H}+\mathrm{Al}=2,82 \mathrm{cmol}(\mathrm{c}) \mathrm{L}^{-1}$; $\mathrm{CTC}_{\text {efetiva }}=13,19$; Saturação de bases $(\mathrm{V})=78,6 \%$. Após correção de solo, as parcelas de $2 \mathrm{~m} \times 4 \mathrm{~m}$ foram demarcadas e realizou-se um corte de uniformização em toda a área experimental. As parcelas foram subdivididas em 04 subparcelas de 0,5 $\mathrm{m} \times 1 \mathrm{~m}$ cada. Desse modo, a área experimental totalizou $24 \mathrm{~m}^{2}$. Com base em uma expectativa de produção de 22 ton $\mathrm{MS} \mathrm{ano}^{-1}$, foram testados 03 (três) tratamentos (quantidades de $\mathrm{N}$, na forma de ureia): dose correta (391 kg de N.ha.ano-1 ${ }^{-1}$, metade (meia) e dobro da dose. A referência para este cálculo e para a correção foram as recomendações do novo manual de adubação e calagem para os Estados do Rio Grande do Sul e de Santa Catarina (CQFS-RS/SC, 2016). Dada a possibilidade de realizar, na região do experimento, aproximadamente 10 pastejos (cortes) por ano na espécie estudada, foi aplicada a décima parte da dose anual de ureia, seguindo sugestões de Pereira et al. (2018) de realizar adubação nitrogenada após cada pastejo em sistemas com alto nível de intensificação. Dada a uniformidade das condições da área, o delineamento experimental foi o inteiramente casualizado, com quatro repetições (subparcelas) em cada tratamento.

Avaliou-se os seguintes parâmetros: Produção de massa verde e massa seca, teor de matéria seca, taxa de acúmulo de forragem e capacidade de suporte. Previamente foi realizado um corte de uniformização e, imediatamente, aplicou-se o $\mathrm{N}$ conforme os tratamentos. As amostragens foram realizadas 33 dias depois, quando a pastagem atingiu altura média de 25 a $28 \mathrm{~cm}$, cortando-se o pasto a uma altura de $10 \mathrm{~cm}$ do solo, fazendo quatro amostragens de $0,25 \mathrm{~m}^{2}$ por tratamento, no centro de cada subparcela, com auxílio de um quadrado de peças de PVC 
encaixadas medindo 0,5 X 0,5 m, para delimitar a área a ser cortada em cada amostragem. O pasto verde foi pesado imediatamente com balança eletrônica à bateria (precisão 0,1 g). Retirou-se $100 \mathrm{~g}$ de cada amostra para secagem em forno micro-ondas, conforme metodologia descrita por Souza et al. (2002), até obtenção de peso constante.

A produção de MV (massa verde) foi obtida pela seguinte fórmula:

$$
P M V=P P V x 10.000
$$

\section{Em que:}

$\mathrm{PMV}=$ Produção de massa verde $\left(\mathrm{kg} \mathrm{ha}^{-1}\right)$

$\mathrm{PPV}=$ Produção de pasto verde $\left(\mathrm{kg} \mathrm{m}^{-2}\right)$

A produção de MS (massa seca) foi obtida pela seguinte fórmula:

$$
P M S=T M S x P M V
$$

\section{Em que:}

PMS = Produção de massa seca $\left(\mathrm{kg} \mathrm{ha}^{-1}\right)$

TMS $=$ Teor de matéria seca $(\%)$

$\mathrm{PMV}=$ Produção de massa verde $\left(\mathrm{kg} \mathrm{ha}^{-1}\right)$

A TAF (taxa de acúmulo de forragem) foi obtida dividindo-se a produção de massa seca pelo período decorrido entre o corte de uniformização e o de amostragem, aplicando-se a seguinte fórmula:

$$
T A F=\frac{P M S}{P E C}
$$

\section{Em que:}

$\mathrm{TAF}=$ Taxa de acúmulo de forragem $\left(\mathrm{kg} \mathrm{MS}\right.$ ha dia $\left.^{-1}\right)$

$\mathrm{PMS}=$ Produção de massa seca $\left(\mathrm{kg} \mathrm{ha}^{-1}\right)$

$\mathrm{PEC}=$ Período entre cortes ( $\mathrm{n}^{\circ}$ de dias)

A CS (capacidade de suporte) foi obtida dividindo-se a produção de massa seca pelo consumo de pasto esperado para uma unidade animal (3,5\% do seu peso vivo) por um período médio entre pastejos estimado em 30 dias, aplicando-se a seguinte fórmula: 


$$
C S=\frac{P M S}{((450 \times 0,035) \times P E P)}
$$

\section{Em que:}

$\mathrm{CS}=$ Capacidade de suporte $(\mathrm{UA} \mathrm{ha-1})$

$\mathrm{PMS}=$ Produção de massa seca $\left(\mathrm{kg} \mathrm{ha}^{-1}\right)$

PEP = Período médio estimado entre pastejos ( $\mathrm{n}^{\circ}$ de dias)

Os dados foram testados quanto à homogeneidade e normalidade (teste de Shapiro-Wilk), seguidos de análise de variância e teste de Tukey, para dados paramétricos, e testes de Kruskal Wallis e Wilcoxon, para os dados nãoparamétricos, com nível de significância de 3\%, no programa $R$ (R DEVELOPMENT CORE TEAM, 2020).

\section{RESULTADOS}

Os dados apresentaram distribuição normal, exceto os de MV (massa verde), que foram analisados com técnicas não paramétricas.

A produção de massa verde, massa seca e o teor de matéria seca (\%) foram influenciados pela dose de N. A produção de MV foi estatisticamente diferente entre todos os tratamentos, correspondendo a $8.304 \mathrm{~kg} \mathrm{ha}^{-1}, 11.376 \mathrm{~kg} \mathrm{ha}^{-1}$ e $8.976 \mathrm{~kg} \mathrm{ha}$ 1 para os tratamentos "meia", "correta" e "dobro", respectivamente. Com relação à MS, na dose "correta", obteve-se $2.287 \mathrm{~kg} \mathrm{MS} \mathrm{ha-1}^{-1}$, sendo estatisticamente superior aos tratamentos "meia" e "dobro", que se mostraram estatisticamente iguais entre si, com $2.001 \mathrm{~kg} \mathrm{MS} \mathrm{ha}^{-1}$ e $1.902 \mathrm{~kg} \mathrm{MS} \mathrm{ha}^{-1}$, respectivamente. O teor de matéria seca foi maior no tratamento "meia" $(24,1 \%)$ enquanto os demais tratamentos não diferiram entre si. Mesmo tendo um teor menor de matéria seca quando comparado ao tratamento restritivo (meia) e estatisticamente igual ao tratamento de excesso (dobro), o uso da dose correta de $\mathrm{N}$ se mostrou vantajoso do ponto de vista produtivo - MV e MS (figura 1). 


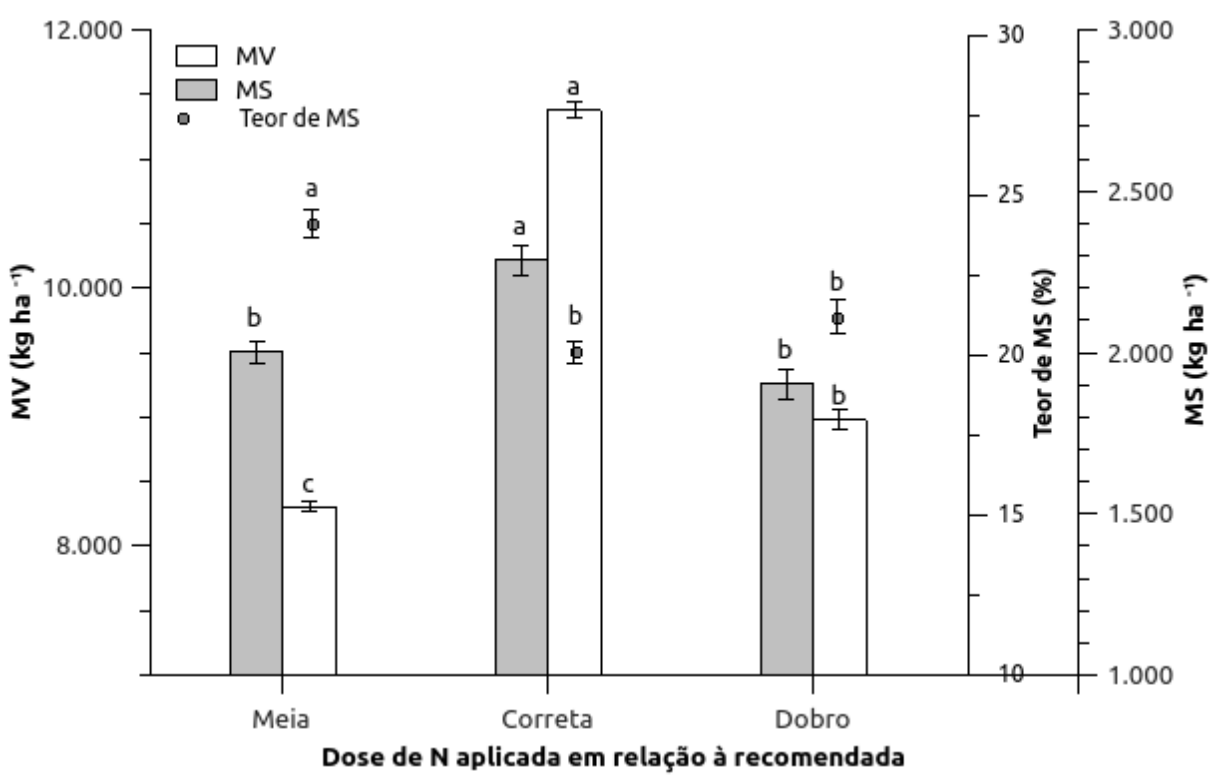

Figura 1. Produção de massa verde, massa seca e teor de matéria seca em pastagem de Tifton 85, de acordo com a quantidade de $\mathrm{N}$ aplicada em relação à dose recomendada. Médias com a mesma letra não diferem pelo teste de Tukey (MS) e pelo teste de Wilcoxon (MV) a $3 \%$.

Figure 1. Production of green mass, dry mass and dry matter content in Tifton 85 pasture, according to the amount of $\mathrm{N}$ applied in relation to the recommended dose. Averages with the same letter do not differ by the

Tukey test (MS) and by the Wilcoxon test (MV) at $3 \%$.

Tanto a taxa de acúmulo de forragem como a capacidade de suporte da pastagem foram influenciadas pelos tratamentos, sendo maiores na dose "correta" e estatisticamente iguais nos tratamentos "meia" e "dobro" (figura 2). A TAF em "correta" foi de 69,3 kg ha dia-1; 60,6 kg ha dia-1 em "meia" e 57,65 kg ha dia-1 em "dobro". A CS, por sua vez, ficou em 4,84 UA ha-1 na dose "correta"; 4,24 UA ha-1 no tratamento "meia" e 4,03 UA ha-1 no tratamento "dobro". 


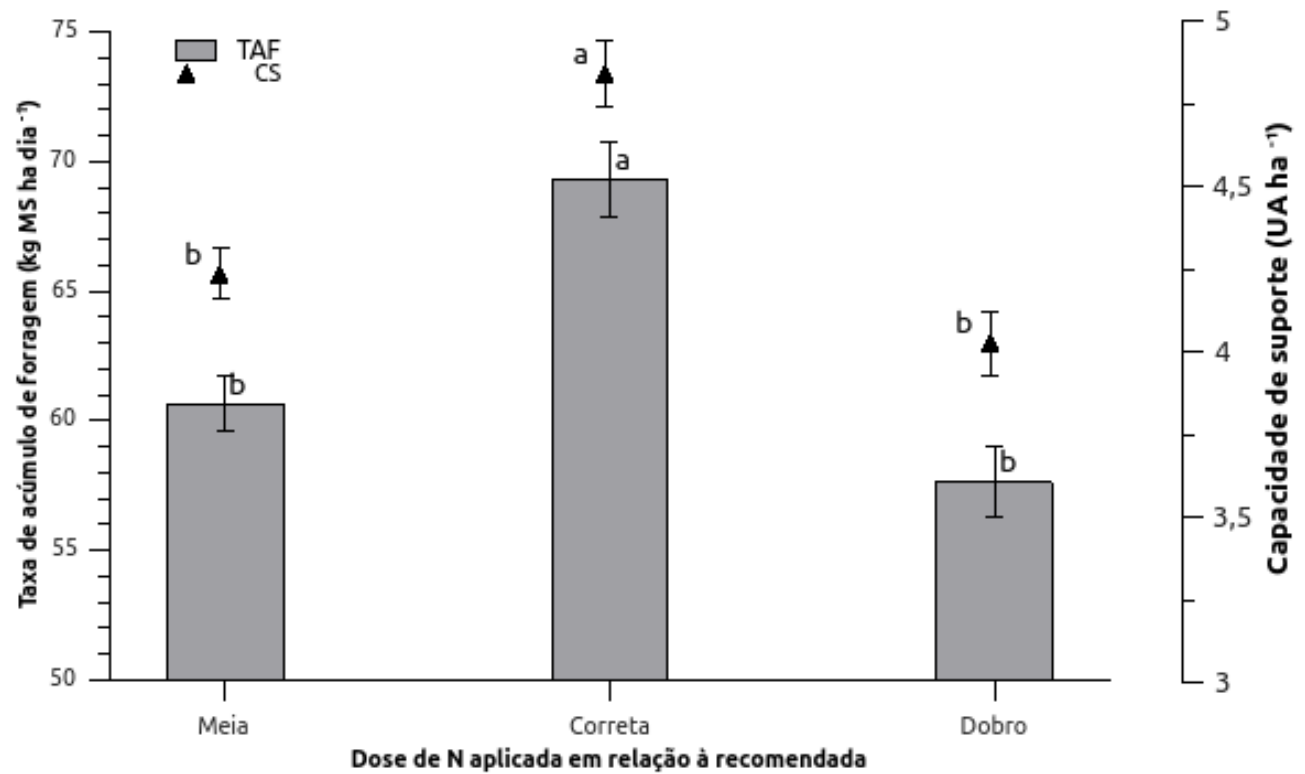

Figura 2. Taxa de acúmulo de forragem e capacidade de suporte em pastagem de Tifton 85, de acordo com a quantidade de $\mathrm{N}$ aplicada em relação à dose recomendada. Médias com a mesma letra não diferem pelo teste de Tukey a $3 \%$.

Figure 2. Forage accumulation rate and carrying capacity in Tifton 85 pasture, according to the amount of $\mathrm{N}$ applied in relation to the recommended dose. Averages with the same letter do not differ by the Tukey test at $3 \%$.

\section{DISCUSSÃO}

Premazzi \& Monteiro (2002) afirmam que o capim Tifton 85 apresenta aumento de produção de massa seca e é responsivo à adubação nitrogenada. Nesse sentido, Oliveira et al. (2011), analisando a produção do capim-coastcrooss, uma forrageira do mesmo gênero do Tifton 85 , sob doses de nitrogênio, obtiveram incrementos que variaram de 7,4 a 10,02 ton ha-1 em seis cortes. Há também outros estudos que testaram doses crescentes de $\mathrm{N}$ mostrando aumentos, geralmente lineares, de produção (Alvim et al., 1999; Ribeiro \& Pereira 2011; Quaresma et al., 2011) . Entretanto, a metodologia usada nos estudos não previu determinar, com base na análise de solo, qual seria a dose adequada de $\mathrm{N}$ para aquela situação específica, não permitindo concluir se as respostas obtidas se deram em razão de uma nutrição adequada ou não.

É amplamente conhecido que a deficiência de $\mathrm{N}$ nas plantas reflete em menor crescimento. $\mathrm{O}$ excesso de $\mathrm{N}$, por outro lado, acelera o crescimento e encurta o Revista Científica Rural, Bagé-RS, volume 22, n2, ano 2020 Submetido 16/06/2020. Aceito 16/10/2020. Doi: https://doi.org/10.30945/rcr-v22i2.3256 
ciclo. Porém, quando o excesso chega a níveis tóxicos (estresse osmótico, ou ainda, estresse salino), as plantas em geral responderão com redução do crescimento, já reportado por Durán Zuazo et al. (2004), explicando porque o tratamento "dobro" apresentou menor produção de MV e MS.

A taxa de acúmulo de forragem é uma importante referência do crescimento das pastagens, enquanto a capacidade de suporte é igualmente relevante, pois permite planejar o ajuste do manejo das pastagens. Esses dois indicadores são influenciados diretamente pela produção de massa seca, que é dividendo em ambas as fórmulas de cálculo. Portanto, é esperado que o tratamento superior em produção mostre-se superior, também, nesses dois parâmetros. Barbero et al. (2009) reportaram resultados de TAF próximos aos desse estudo com coastcross e, assim como Castagnara et al. (2011), também observaram TAF maior nos tratamentos mais produtivos, corroborando com nossos resultados.

\section{CONCLUSÕES}

- O uso da dose correta de $\mathrm{N}$ proporciona maior produção de massa verde, massa seca, maior taxa de acúmulo de forragem e maior capacidade de suporte;

- O tratamento com restrição de $\mathrm{N}$ resulta em maior teor de matéria seca.

\section{AGRADECIMENTOS}

Aos proprietários Elizandro e Eluana Contini, por ceder a área, pelo apoio na realização das atividades e pela confiança.

\section{REFERÊNCIAS}

ALVIM, M. J.; XAVIER, D. S.;.BOTREL, M. de A; MARTINS, C. E.. Resposta do coast-cross (Cynodon dactylon (L.) Pers.) a diferentes doses de nitrogênio e intervalos de cortes. Revista Brasileira de Zootecnia, v. 27, n. 5, p. 833-840, 1998. 
ALVIM, M. J.; XAVIER, D. S.; VERNEQUE, R. da S.;.BOTREL, M. de A. Resposta do tifton 85 a doses de nitrogênio e intervalos de cortes. Pesquisa Agropecuária Brasileira, Brasília, v.34, n.12, p.23452352, dez. 1999.

BARBERO, L. M.; CECATO, U.; LUGÃO, S. M. B.; GOMES, J. A. N.; LIMÃO, V. A.; BASSO, K. C. Produção de forragem e componentes morfológicos em pastagem de coastcross consorciada com amendoim forrageiro. Revista Brasileira de Zootecnia, v. 38, n. 5, p. 788-785, 2009.

CASTAGNARA, D. D. ; ZOZ, T.; KRUTZMANN, A.; UHLEIN, A.; MESQUITA, E. E.; NERES, M. A.; RABELLO DE OLIVEIRA, P. S. Produção de forragem, características estruturais e eficiência de utilização do nitrogênio em forrageiras tropicais sob adubação nitrogenada. Semina: Ciências Agrárias, v. 32, n. 4, p. 1637-1648, 2011.

DURAN ZUAZO, V. H.; RAYA, A. M.; FRANCO, J. A. R. D. Impact of salinity on macro- and micronutrients uptake in mango (Mangifera indica L. cv. Osteen) with different rootstocks. Spanish Journal of Agricultural Research, v. 2, n. 1, p. 121, 2004.

FAGUNDES, J. L.; MOREIRA, A. L.; FREITAS, A. W. De P.; ZONTA, A.; HEINRICHS, R.; ROCHA, F. C.; BACKES, A. A.; VIEIRA, J. S. Capacidade de suporte de pastagens de capim-tifton 85 adubado com nitrogênio manejadas em lotação contínua com ovinos. Revista Brasileira de Zootecnia, v. 40, n. 12, p. 2651-2657, 2011.

HILL, G. M.; GATES, R. N.; WEST, J. W.; BURTON, G. W. Tifton 85 bermudagrass utilization in beef, dairy, and hay production. In: WORKSHOPSOBRE O POTENCIAL FORRAGEIRO DO GÊNERO CYNODON, 1996, Juiz deFora. Anais... Juiz de Fora: Embrapa-CNPGL, 1996. p. 139-150. 
IBGE, I. B. de G. e E. Pesquisa Trimestral do Leite. Disponível em: <http://www.sidra.ibge.gov.br/bda/tabela/listabl.asp?c=1086\&z=t\&o=24>. Acesso em: 8 abr. 2020.

INMET. INMET - Instituto Nacional de Meteorologia, 2020. . Disponível em: <http://www.inmet.gov.br/portal/index.php?r=clima/normaisclimatologicas $>$. Acesso em: 13 abr. 2020.

Manual de adubação e calagem para os Estados do Rio Grande do Sul e de Santa Catarina. 11. ed. Porto Alegre: Sociedade Brasileira de Ciência do Solo, Núcleo Regional Sul, Comissão de Química e Fertilidade do Solo RS/SC, 2016.

OLIVEIRA, M. A.; PEREIRA, O.G.; RIBEIRO, K. G.; SANTOS, M. E. R.; CHIZZOTTI, F. H. M.; CECON, P. R. Production and nutritional value of bermudagrass cv. coastcross grown under different nitrogen doses and regrowth ages. Arquivo Brasileiro de Medicina Veterinária e Zootecnia, v. 63, n. 3, p. 694-703, 2011.

PATZLAFF, N. L.; MARTINS, C. E. N.; ARBOITTE, M. Z.; HÖFS, A. Variedades de milho com polinização aberta da Epagri sob efeito do espaçamento entre linhas. Brazilian Journal of Development, v. 6, n. 2, p. 5750-5766, 4 fev. 2020.

PEDREIRA, C. G. S. Avaliação de novas gramíneas do gênero Cynodon para a pecuária do sudeste dos Estados Unidos. In: WORKSHOP SOBRE O POTENCIAL FORRAGEIRO DO GÊNERO CYNODON, Juiz de Fora. Anais... Juiz de Fora: Embrapa-CNPGL, 1996.

PEREIRA, L. E. T.; NISHIDA, N. T.; CARVALHO, L. da R.; HERLING, V. R. Recomendações para correção e adubação de pastagens tropicais. Pirassununga: Faculdade de Zootecnia e Engenharia de Alimentos da USP, 2018.

PREMAZZI, L. M. \& MONTEIRO, F. A. Produção do capim-Tifton 85 submetido a doses e épocas de aplicação de nitrogênio após o corte. Boletim de Indústria Animal, v. 59, p. 1-16, 2002. 
QUARESMA, J. P. de S.; ALMEIDA, R. G. de; ABREU; Abreu, J. G. de; CABRAL, L. da S., OLIVEIRA, M. A. de; CARVALHO, D. M. G. De. Produção e composição bromatológica do capim-tifton 85 (Cynodon spp.) submetido a doses de nitrogênio. A Acta Scientiarum. Animal Sciences, Maringá , v. 33, n. 2, p. 145-150, 2011. Disponível em <http://www.scielo.br/scielo.php?script=sci_arttext\&pid=S1807$86722011000200005 \&$ Ing=en\&nrm=iso >. Acesso em: 12 out. 2020.

R DEVELOPMENT CORE TEAM. R: A Language and Environment for Statistical Computing R Foundation for Statistical Computing. Vienna, Austria, 2020.

RIBEIRO, K. G. \& PEREIRA, O. G. Produtividade de matéria seca e composição mineral do capim-tifton 85 sob diferentes doses de nitrogênio e idades de rebrotação. Ciência e Agrotecnologia, Lavras, v. 35, n. 4, p. 811-816, 2011. DOI: https://dx.doi.org/10.1590/S1413-70542011000400022. Acesso em: 12 out. 2020

SOUZA, G. B. de; NOGUEIRA, A. R. D. A.; RASSINI, J. B. Determinação de matéria seca e umidade em solos e plantas com forno de microondas doméstico. Circular Técnica MAPA, v. 33, p. 1-9, 2002. 